Onkologe 2014 · 20:310-311

DOI 10.1007/s00761-013-2537-6

Online publiziert: 26. März 2014

(c) Springer-Verlag Berlin Heidelberg 2014

M.W. Beckmann ${ }^{1}$ - S. Hegewisch-Becker ${ }^{2}$ - M. Bamberg ${ }^{3}$

${ }^{1}$ Frauenklinik, Universitätsklinikum Erlangen

2 Onkologische Schwerpunktpraxis Hamburg

${ }^{3}$ Universitätsklinikum Tübingen

\title{
Vulva- und Vaginalkarzinome
}

Liebe Kolleginnen und Kollegen,

wir freuen uns, Ihnen mit dem Heft von Der Onkologe zum Thema „Vulva und Vagina" zwei seltene Tumorentitäten der gynäkologischen Tumoren vorstellen zu dürfen.Während die Inzidenz des Vaginalkarzinoms in den letzten 10 Jahren gleich geblieben ist, steigt die Inzidenz des Vulvakarzinoms kontinuierlich an und hat sich in den letzten 15 Jahren nach Hochrechnungen aus einigen Klinischen Krebsregistern fast verdoppelt. Dies hat zu einer anderen Klassifikation und auch weiteren Untersuchungen zur Ätiologie geführt, die die Humane-Papilloma-Viren-Infektion - ähnlich wie beim Zervixkarzinom - als eine der Ursachen des vermehrten Auftretens der Vulvakarzinome identifiziert haben.

\section{》) Die Inzidenz des Vulvakarzinoms steigt kontinuierlich an}

Die ansteigende Inzidenz eines Karzinoms der älteren Frau Ende der 1960er-/ Anfang der 1970er-Lebensdekade geht, bedingt durch die altersspezifischen Komorbiditäten, mit Einschränkung der operativen Radikalität, der Strahlentherapie oder der medikamentösen Therapien einher. Somit ist es nicht verwunderlich, dass das Sentinel-Konzept, eingeschränkte partielle radikale Operationen oder die fokussierte Radiatio in der Diskussion sind - mit dem Ziel, die therapieassoziierte Morbidität der Patientinnen zu reduzieren. Die engmaschige Nachsorge ist Voraussetzung dafür, bei frühem Rezi- div oder erneutem Auftreten von Vorstufen eine minimale Reintervention zu ermöglichen.

Wir hoffen, dass wir Ihnen mit dieser Zusammenstellung des Hefts zu Tumoren von Vulva und Vagina eine umfassende Übersicht über die aktuellen diagnostischen und therapeutischen Konzepte vorstellen können.

Für die Schriftleiter des Schwerpunktthemenhefts

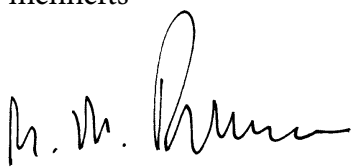

Matthias W. Beckmann

Für die Herausgeber
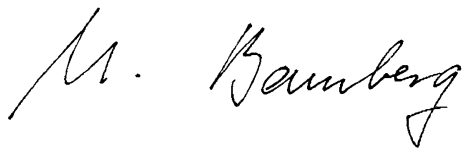

Michael Bamberg

\section{Korrespondenzadresse}

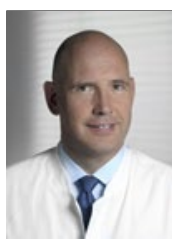

Prof. Dr. M.W. Beckmann

Frauenklinik, Universitätsklinikum Erlangen

Universitätsstraße 21-23,

91054 Erlangen

fk-direktion@uk-erlangen.de

\section{Einhaltung der ethischen Richtlinien}

Interessenkonflikt. M.W. Beckmann, S. HegewischBecker und M. Bamberg geben an, dass kein Interessenkonflikt besteht. 
Hier steht eine Anzeige.

黛 Springer 